Bull. Mater. Sci., Vol, 10, Nos 1\& 2, March 1988, pp. 75-76. (C) Printed in India.

\title{
The nature of dislocation motion in quasicrystals
}

\author{
SRIRAM RAMASWAMY \\ Department of Physics. Indian Institute of Science, Bangalore 560012, India.
}

Abstract. Calculations in a hydrodynamic model of quasicrystal dynamics show that dislocation motion in these systems is impeded by a drag far greater than that in crystals.

Keywords. Dislocation motion; quasicrystal.

This work was done while the author was at the University of Pennsylvania. The details are already in print (Lubensky et al 1985, 1986; see also Levine et al 1985). Only a brief summary will be given here.

Our approach to the problem consisted of three steps. First, we answered the question, "what is a quasicrystal dislocation?". Next we derived the equations of hydrodynamics for the long-wavelength, low-frequency excitations of a quasicrystal. And finally we used these equations along with the definition of a dislocation to understand the processes involved in moving a dislocation through a quasicrystal. The conclusion of this study was that--as an inevitable consequence of the absence of periodic crystalline order - a dislocation moving in any direction in a quasicrystal feels a drag force of the same order of magnitude as that felt by a dislocation moving in a climb direction in a conventional periodic crystal. This effectively rules out plastic deformation: quasicrystals, even if nearly perfect, should be exceedingly brittle. We present qualitative arguments to make our results plausible. The detailed derivation is given in Lubensky et al (1986). The techniques used are those of Dubois-Violette et al (1983).

The crucial difference between a three-dimensional crystal and an icosahedral quasicrystal, for our purposes, is that since the reciprocal lattice of the latter has six basis vectors, its elastic theory and long wavelength dynamics involve six brokensymmetry variables: a three-component "phonon" displacement field $u$, as in a crystal, and another three-component field $w$, called a "phason". The phason variables describe internal rearrangements, and thus, even at zero wave-vector, involve relative motion of atoms. They are thus diffusive, with a diffusion constant of the same order as that for vacancies (about $10^{-11} \mathrm{~cm}^{2} / \mathrm{sec}$ ), which means they relax exceedingly slowly. Dislocations are defined by a natural generalization of their definition in crystals. However, the quasiperiodic nature of the system also forces every dislocation to have a $u$-part and a $w$-part (if it had only a $u$-part, a simple analysis of a Burgers circuit would show that the system must be periodic). Thus, a moving dislocation must drag along a slow and unresponsive $w$-field. It is precisely as if the dislocation were always moving in a climb direction, since its motion requires the constant rearrangement of matter far from the core. It should therefore be no surprise that the mobility of a dislocation in these materials turns out to be

$$
M_{q} \sim D / K a^{2} \sim\left(10^{8} \text { poise }\right)^{-1}
$$

where $D$ is a phason diffusion constant (of order vacancy diffusion constants in 
crystals), $K$ is an elastic constant, and $a$ is a unit cell size. By contrast, the mobility for glide in periodic crystals is

$$
M_{c} \sim \eta^{-1} \sim(1 \text { poise })^{-1}
$$

where $\eta$ is a "crystal viscosity", i.e. a sound damping coefficient.

The main consequence of this, as discussed earlier, is that plastic deformation should be practically impossible. It also probably means that the process of annealing out phason and dislocation strains in quasicrystals should be very slow, and that therefore even large single grains should have short translational correlation lengths. This seems to be in accord with recent experiments (Horn et al 1987).

\section{References}

Dubois-Violette E, Guazzelli E and Prost J 1983 Philos. Mag. A48 727

Horn P M, Heiney P A, Bancel P A, Gayle F W, Jordan J L, LaPlaca S and Angilello J 1987 Science (to appear)

Levine D, Lubensky T C, Ostlund S, Ramaswamy S, Steinhardt P J and Toner J 1985 Phys. Rev. Lett. 54 1520

Lubensky T C, Ramaswamy S and Toner J 1985 Phys. Rev. B32 7444

Lubensky T C, Ramaswamy S and Toner J 1986 Phys. Rev. B33 7715 